\title{
On the Extent to which Coherent Bremsstrahlung from Crystals Can Be Monochromatized.
}

\author{
G. Bolonina \\ Laboratori Nazionali del CNEN - Frascati \\ (Nuove Gimento, $49 \wedge, 756(1967))$
}

(1) is the number of the second only of the two equations on top of page 758 . Please, take out the curly bracket in front of the two equations.

The first of eqs. (8) should read

$$
\Phi_{1}=\sum_{g} \frac{\Phi\left(g^{2}\right)}{Q_{z}^{2}}
$$

The third of eqs. (12) should read

$$
\chi_{3}(x)=-2 \pi D^{2} \sum_{g} \frac{\Phi\left(g^{2}\right)}{Q_{z}^{1}} \cos 2 \varphi
$$

In the 6th line before eqs. (15): where should be replaced by were.

The first of eqs. (15) should read

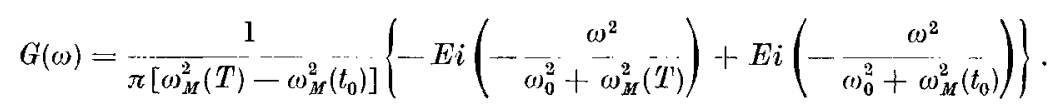

In Fig. 2, along the vertical axis, protons should be replaced by photons. 